The EHRs did not record RF evolution, TDM requests and results or PIs in 53 (70\%), 23 (30\%), $39(51 \%)$ and 61 (80\%) cases respectively.

OR for recorded TDM results related to highly important PIs compared to low-importance PIs, for recorded TDM ordering related to moderate RI compared to normal RF and records for RF evolution related to moderate RI compared to normal RF were 3 (95\%CI: 1-9; $\mathrm{p}=0.046), \quad 0.3(95 \% \mathrm{CI}: 0.8-0.9 ; \quad \mathrm{p}=0.04)$ and $4(95 \% \mathrm{CI}: 1-16$; $p=0.029)$, respectively. A significant linear trend was observed. OR for all other variables was non-significant.

Conclusions The low percentage recording of TDM-related variables and pharmacist interventions in EHR potentially limits interprofessional communication and the decision-making process. This fact highlights the need for clinical pharmacists to safeguard the information they have discovered by recording their interventions in the EHR as a clinical episode in comprehensive patient care. This will increase the visibility of the pharmacist and the effect of his/ her actions.

No conflict of interest.

\section{TCH-049 TOPICAL MORPHINE GELS FOR PAINFUL WOUNDS}

\section{doi:10.1136/ejhpharm-2013-000276.240}

'D Mateus, 'P Trindade, ${ }^{1} \mathrm{H}$ Gonçalves, ${ }^{2} \mathrm{~A}$ Salgado, ${ }^{2} \mathrm{~J}$ Marto, ${ }^{3 P}$ Machado, ${ }^{1} \mathrm{~A}$ Melo Gouveia, ${ }^{2} \mathrm{HM}$ Ribeiro, ${ }^{2} \mathrm{~A}$ Almeida. 'Instituto Português de Lisboa Francisco Gentil EPE, Pharmacy, Lisbon, Portugal; 'iMed.UL - Research Institute for Medicines and Pharmaceutical Sciences FFUL, Nanomedicine and Drug Delivery Systems, Lisbon, Portugal; ' ${ }^{\text {Faculty }}$ of Pharmacy-University of Lisbon, Microbiology, Lisbon, Portugal

Background The use of morphine applied topically to painful wounds has potential advantages such as a lower dose than with systemic administration and fewer side effects. Gels are known to be suitable for treating wounds.

Purpose To develop two physicochemically and microbiologically stable gels: a more viscous formulation (F1) and a fluid formulation for spraying (F2), both containing morphine hydrochloride $(\mathrm{MH})$. The effect of viscosity on drug release from both gels was also investigated.

Materials and Methods Sodium carboxymethylcellulose-based aqueous gels were prepared and sterilised by autoclaving. The $0.125 \% \mathrm{w} / \mathrm{w}(\mathrm{F} 1)$ and $1.0 \% \mathrm{w} / \mathrm{w}$ (F2) gels containing $\mathrm{MH}$ were compounded using an injectable solution of $\mathrm{MH}$ and preservatives (parabens). Preparation and primary packaging were performed inside a horizontal laminar flow hood. Primary packaging consisted of single dose syringes for $\mathrm{F} 1$ and $10 \mathrm{~mL}$ amber glass bottles with pump sprays for F2. Stability studies were performed using 3 batches of each final formulation. Samples were stored at $5 \pm 3^{\circ} \mathrm{C}$, at $22 \pm 3^{\circ} \mathrm{C}$ (light exposed and protected) and $40 \pm 2^{\circ} \mathrm{C} / 75 \pm 5 \%$ $\mathrm{RH}$ for 98 days (samples collected at 6 time points). Organoleptic characteristics, $\mathrm{pH}$, viscosity, $\mathrm{MH}$ and preservative content were assessed. Sterility tests, microbiological control and preservative efficacy were studied according to $\mathrm{Ph}$. Eur. The $\mathrm{MH}$ release profile was evaluated using Franz cells.

Results Formulations were odourless, yellowish, translucent and homogeneous. The $\mathrm{pH}$ was 6.35 (F1) and 5.70 (F2), viscosity was $52.933 \mathrm{mPa}$.s at $6.12 \mathrm{~s}-1$ (F1) and $16.7 \mathrm{mPa}$.s at $12.24 \mathrm{~s}-1$ (F2). Methylparaben, propylparaben and $\mathrm{MH}$ contents were between $90-110 \%$. Preservatives were effective and preparations remained sterile and stable for 60 days. $\mathrm{MH}$ release was slow and inversely proportional to viscosity.

Conclusions The MH gels presented suitable physicochemical and pharmaceutical characteristics for topical application to painful wounds. The slow release profile may reduce the number of applications.

No conflict of interest.

\section{TCH-050 USE OF ELECTROENCEPHALOGRAPHY (EEG)-BASED METRICS TO TEST THE GUSTATORY PROPERTIES OF LIQUID TRIMETHOPRIM FORMULATIONS}

doi:10.1136/ejhpharm-2013-000276.241

${ }^{1 P}$ Almeida, ${ }^{1 P}$ Chaves, ${ }^{2} \mathrm{P}$ Carinha, ${ }^{3} \mathrm{P}$ Barata, ${ }^{4} \mathrm{D}$ Santos. ${ }^{1}$ University of Porto, Laboratory of Neuropsychophysiology, Porto, Portugal; '2Centro Hospital S Joao, Serviços Farmacêuticos, Porto, Portugal; ${ }^{3}$ Faculdade Ciências da Saúde, Universidade Fernando Pessoa, Porto, Portugal; ' ${ }^{4}$ aculdade Farmácia, Universidade Porto, Porto, Portugal

Background It is well known that the gustatory properties of a formulation strongly affect patient adherence to a treatment. However measuring these properties is highly subjective and difficult, especially for the paediatric population. The use of neuropsychophysiological indexes and covert behaviours in assessing the attractive properties of sensorial stimuli has a long tradition in the domain of affective neuroscience. Ways of measuring range from the use of autonomous nervous system activation patterns, to features extracted from electroencephalographic activity or simple and discriminative reaction time tasks. These measurements provide alternative means for assessing the characteristics of commercial products, overcoming the limitations of self reporting-based research, namely social desirability, and for studying populations unable to provide usable verbal responses (e.g. children).

Purpose To find out if this methodology can be used for evaluating the gustatory properties of formulations in order to enhance patient adherence.

Materials and Methods Trimethoprim formulations were prepared using NF syrup. Flavour was added afterwards. Participants were stimulated with 3 different flavoured formulations (banana, red berry and neutral) for 10 seconds each while subjected to an EEG recording. The order of presentation was fully counter-balanced between subjects. Subjects rated the different solutions for palatability and intensity. Five seconds of the EEG response for each sample were converted to the frequency domain, and the log power and inter-hemispheric asymmetry were calculated for anterior, central and parietal electrodes. Different algorithms, combining different EEG features, were tested for predictive power regarding palatability and type of formula

Results Theta inter-hemispheric activity at parietal electrodes predicted the behavioural assessment of palatability $(\mathrm{R} 2=0.35)$. Moreover, the application of unsupervised learning methods, such as Support Vector Machines, on the log power at different bands from 0 to $12 \mathrm{~Hz}$, could distinguish with up to $95.24 \%$ accuracy between flavoured and non-altered solutions.

Conclusions This technology can be used in formulation studies that are attempting to enhance the organoleptic properties of a formulation.

No conflict of interest.

\section{TCH-051 VALIDATION OF AN AUTOMATED COMPOUNDER SET UP ONCE A WEEK FOR PARENTERAL NUTRITION SOLUTIONS}

doi:10.1136/ejhpharm-2013-000276.242

L Bouchoud, F Sadeghipour, S Fleury, P Bonnabry. University Hospitals of Geneva, Pharmacy, Geneva, Switzerland

Background Our parenteral nutrition production (PN) decreased after we introduced standard solutions. To keep just a small number of daily PN items cost-effective, we decided to validate a once a week setting up of an automated compounder device (ACD).

Purpose To test the operation and performance of an ACD (Baxa MM12) for a once a day and a once a week use.

Materials and Methods Accuracy (mean in \% of the expected value) and precision (Coefficient of Variation) of the ACD was evaluated by weighing different volumes of water 10 times ( 0.5 to $40 \mathrm{~mL}$; daily operational qualification) and different volumes of 